\title{
Kooperationsprozesse und Echtzeitmanagement
}

\author{
Enrico Senger, Hubert Österle \\ Institut für Wirtschaftsinformatik, Universität St. Gallen
}

\section{Auslöser und Veränderungen}

Das Unternehmen des Informationszeitalters ist nicht mehr produkt-, sondern kundenorientiert. Es muss das Kundenproblem in seinem vollen Umfang verstehen und dem Kunden so viele zusammenhängende Teilprobleme wie möglich abnehmen. Ausgangspunkt ist folglich der Kundenprozess, d.h. die Abfolge von Aufgaben, die der Kunde bei Problemlösungen ausführt. Der Kundenprozess bestimmt den Bedarf an Produkten und Dienstleistungen, die der Lieferant anbieten kann oder muss (s. dazu Porter u. Millar 1985; Ives u. Learmonth 1984; Österle 1995). Gleichzeitig kann ein Unternehmen nur dann wirtschaftlich erfolgreich sein, wenn es sich auf die Erstellung der Leistungen konzentriert, für die es über ein herausragendes, wettbewerbsfähiges Know-how verfügt (Kernkompetenz) und alle anderen Leistungen zukauft, die Dritte besser, schneller und billiger erbringen können (vgl. Prahalad u. Hamel 1990). Das Ergebnis sind Geschäftsnetzwerke (Wertschöpfungsnetze, Supply Chains), in denen alle Beteiligten ein Kundenproblem schnell und umfassend lösen. Voraussetzung dafür ist die Integration bislang weitgehend voneinander unabhängiger interner Prozessschritte der Beteiligten über gemeinsam definierte und betriebene Kooperationsprozesse.

Die Informationstechnologie ist der Enabler, d.h. sie schafft die notwendigen Voraussetzungen für Kooperationsprozesse, indem sie Informationen miteinander verknüpft und vom Entstehungsort (Point-of-Creation) zum Verwendungsort (Point-of-Action) transportiert, also beispielsweise von der Ladenkasse zu allen vorgelagerten Stufen der Supply Chain (z. B. zum Warenverteiler, zum Produzenten, zur Bank usw.). Der gesamte dem Verkauf vorgelagerte Prozess kann umso besser funktionieren, je früher alle Beteiligten die relevanten Informationen nutzen können. Das Problem ist dabei weder die elektronische Erfassung der Daten, noch ihre Verarbei- 
tung, sondern die Verbindung (Relationship) zwischen Datenentstehung und Datenverwendung (Integration).

Der vorliegende Beitrag fokussiert auf die Frage, wie Unternehmen ihre Wettbewerbsposition verbessern können, indem sie informationsgestützt über Unternehmensgrenzen hinweg zusammenarbeiten. Eine Fallstudie der Schiesser AG und ihrer Partner illustriert die Potentiale. Die vergleichende Untersuchung ähnlicher Fallstudien führt dann zunächst zu Mustern einer erfolgreichen Transformation und liefert schließlich Anhaltspunkte für Handlungsoptionen.

\section{Reorganisation der Bestelllogistik bei der Schiesser AG1}

\subsection{Unternehmen}

Die Schiesser AG (www.schiesser.com) mit Sitz in Radolfzell (D) und weiteren Produktionsstandorten in Tschechien, Bulgarien und der Slowakei ist der führende deutsche Wäschespezialist und auf dem Weg zum internationalen Marktführer im textilen „close to skin“ - Produktbereich. Der Handel als direkter Kunde legt neben der Produktqualität einen besonderen Wert auf die Lieferpünktlichkeit, um die von kurzlebigen Modetrends beeinflusste Nachfrage des Endverbrauchers zeitnah bedienen zu können. Dies setzt eine pünktliche und kostengünstige Versorgung aller Produktionsstandorte mit den benötigten Rohmaterialien voraus.

\subsection{Ausgangssituation}

Schiesser plante die Produktion zentral für alle Produktionsstätten. Etwa $70 \%$ der Waren gingen zunächst in das zentrale Beschaffungszentrum in Radolfszell. Schiesser teilte dort die Lieferungen auf die verschiedenen Produktionsstätten auf und versandte sie neu gebündelt mit unterschiedlichen Spediteuren an die einzelnen Werke. Die Zentrale bezahlte die Rechnungen der Lieferanten und verrechnete sie anschließend mit den Töchtern. Dieser Beschaffungsprozess war zeitaufwändig und teuer:

- Da die Ware frei Haus nach Radolfszell bzw. direkt in die Werke geliefert wurde, beauftragten die Lieferanten eine Vielzahl unterschiedlicher Transportlogistikdienstleister. Schiesser hatte einen hohen Koordinati-

${ }^{1}$ Ausführlich in (Senger u. Österle 2003c) 
onsaufwand und musste laufend überprüfen, ob die Lieferungen rechtzeitig zum Bedarfszeitpunkt im Werk sein konnten. Die Transportkosten lagen über denen bei einer Konzentration auf weniger Transporteure mit größeren Transportmengen.

- Schiesser verzollte die Einkaufswaren selbst, und dies teilweise mehrfach.

- Schwierigkeiten bei der Lieferpünktlichkeit führten zu hohen Sicherheitsbeständen in den Werken. Durch fehlende Transparenz in der Lieferkette war Schiesser nicht in der Lage, die Anzahl problembehafteter Lieferungen und deren Gründe zu benennen.

- Die Abgleichung von Unstimmigkeiten per Telefon und Fax war durch die langen Kommunikationswege sehr zeitaufwändig.

\subsection{Projekt}

Schiesser straffte die Beschaffungsprozesse. Operative Aufgaben wie die Bestellung liegen nun bei den Produktionswerken. Die beschriebenen Transportineffizienzen legten eine zentrale Koordination nahe. Dazu lagerte Schiesser das Management des Beschaffungsprozesses von der Bestellung bis zur Anlieferung im Werk an den international tätigen österreichischen Logistikkonzern „Gebrüder Weiss“ aus. Ziele dieses Optimierungsprojektes waren:

- die pünktliche Versorgung der Produktionsstätten mit Einkaufsgütern,

- reduzierte Personalkosten durch die Auslagerung von Aufgaben aus den Bereichen Transport und Zoll,

- Einsparung von Transport- und Zollkosten durch eine gebündelte Abholung der Ware beim Lieferanten und ein gebündeltes Zustellen des Einkaufsmaterials an die Produktionsstandorte sowie deren vereinfachte Verrechnung und

- eine zentralen Anlaufstelle für alle Beteiligten in der Lieferkette.

\subsection{Neue Lösung}

Gebrüder Weiss steuert nun die gesamte Lieferkette ab der Bereitstellung der Waren beim Lieferanten. So kann der Logistiker ausgehend von den Bedarfszeitpunkten im Werk den Abholtermin beim Lieferanten vorgeben und für die rechtzeitige Zustellung Sorge tragen.

Die Produktionsstätten bestellen elektronisch über eine gemeinsame Plattform, die durch den Application Service Provider inet-logistics bereitgestellt wird. Der Logistiker transportiert die bestellte Ware nach ihrer Be- 
reitstellung durch die Lieferanten $\mathrm{zu}$ seinem zentralen Umschlaglager (,Transshipment-Point“), bündelt dort die Lieferungen für die einzelnen Produktionswerke und verzollt sie bei der Ankunft in den Werken.

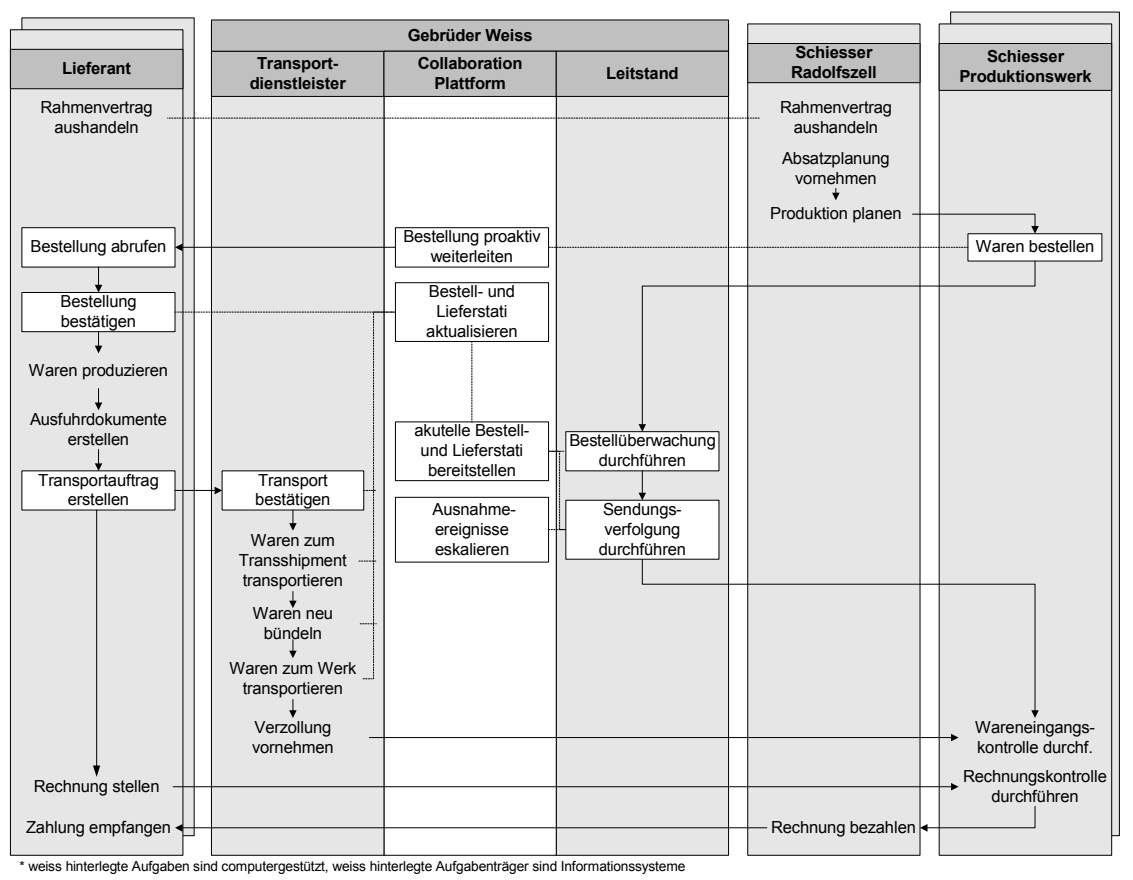

Abb. 1. Kooperationsprozess in der Bestelllogistik von Schiesser

Das internetbasierte System versorgt alle Beteiligten jederzeit mit aktuellen Statusinformationen. Ein Mitarbeiter von Gebrüder Weiss überwacht den Prozess als „Supply Chain Captain“ und greift bei Bedarf steuernd in den Prozess ein.

Pro Jahr werden etwa 12'000 Bestellungen und 3'800 Transporte mit 100 Lieferanten abgewickelt. Schiesser reduzierte durch die neue Lösung die Personalkosten in der Transportlogistik um $85 \%$ und spart jährlich Transportkosten in Höhe von 65'000 EUR. Gebrüder Weiss hat p.a. 54'000 EUR weniger Kosten als bei gleicher Menge herkömmlicher Transportaufträge.

\subsection{Erkenntnisse}

Anstelle interner Prozesse, die voneinander weitgehend unabhängig waren und daher aufwändig koordiniert werden mussten, tritt ein gemeinsam ver- 
einbarter Prozess. Dieser Kooperationsprozess definiert und koordiniert Aufgaben und Zuständigkeiten der Beteiligten über die Unternehmensgrenzen hinweg. Dies schafft Transparenz, ordnet Aufgaben (wie den Warenumschlag) effizienter zu und vermeidet Doppelarbeiten und Liegezeiten. Die erhöhte Prozesseffizienz schafft Zusatznutzen für alle Beteiligten.

Voraussetzung hierfür ist die kontextbasierte unternehmensübergreifende Verknüpfung von Daten (Relationship). Prozesse und Systeme müssen dazu den Orte der Datenentstehung mit dem der Datenverwendung verbinden (Integration). Im Fall Schiesser ermöglicht die gemeinsame Informationsplattform ein erfolgreiches Outsourcing der Transportlogistik. Sie führt die für die Koordination der Lieferkette notwendigen Daten der Schiesser Zentrale, der Produktionswerke, des Logistikers und Lieferanten zeitnah zusammen und bereitet sie adressatengerecht auf.

\section{Fallstudien zum Business Networking}

\subsection{Ziel und Vorgehen}

Ähnlich wie Schiesser und seine Partner haben viele Unternehmen begonnen, Kooperationsprozesse über mehrere Stufen der Wertschöpfungskette zu definieren. Kooperationsprozesse verknüpfen Prozessketten autonomer Geschäftseinheiten unter einer gemeinsamen Prozessführung. Die Partner stimmen ihre Ziele aufeinander ab, um gemeinsam von der gesteigerten Prozesseffizienz zu profitieren.

Derzeit existiert eine Vielzahl von Geschäftsmodellen und Vernetzungskonzepten (z.B. Marktplätze, Portale etc.). Die Auswahl der für ein Unternehmen erfolgsversprechendsten Lösung setzt eine detaillierte Kenntnis der Wettbewerbsposition des Unternehmens und seines wirtschaftlichen Umfeldes ebenso voraus, wie das Wissen um die Potentiale der Informationstechnologie. Diese ganzheitliche Betrachtung des Geschäfts auf den Ebenen Strategie, Prozess und Systeme ist Voraussetzung für eine erfolgreiche Umsetzung (s. dazu Bauer u. Stickel 1998; Österle 1995, Swatman u. Clarke 1990).

Das Institut für Wirtschaftsinformatik der Universität St. Gallen sammelt in Zusammenarbeit mit dem Glassmeyer/McNamee Center for Digital Strategies der Tuck School of Business at Dartmouth Fallstudien erfolgreich eingeführter Lösungen ${ }^{2}$. Ziel ist es, Muster herauszuarbeiten, die auf-

2 Die Arbeit entstand im Rahmen des Forschungsprogramms Business Engineering der Universität St. Gallen (BE HSG) 
zeigen, wie Unternehmen Wettbewerbsvorteile durch den Einsatz von Kooperationsprozessen erzielen können und welche Faktoren dafür erfolgsentscheidend sind. Diese Muster geben den Unternehmen Hinweise zur Nutzung IT-induzierter Veränderungen der Wirtschaft (Handlungsoptionen).

Alle Kooperationsprojekte werden in einer einheitlichen Struktur erhoben und beschrieben (s. Abb. 2). Diese folgt einer für die Untersuchung von Transformationsprojekten von Yin (1994) und Stake (1995) abgeleiteten Fallstudienmethodik (s. Senger u. Österle 2002).

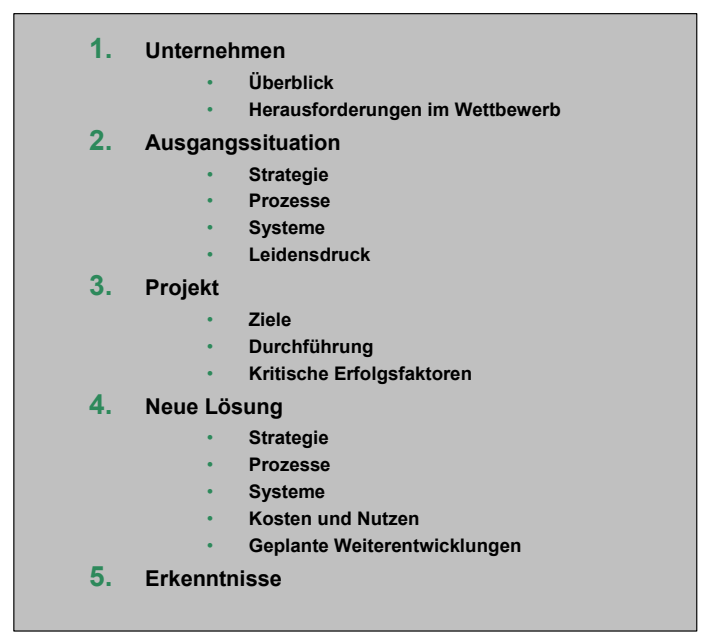

Abb. 2. Strukturierungsrahmen der untersuchten Fälle

Ein wesentliches Betrachtungselement ist dabei der „Leidensdruck“, d.h. die subjektive Problemwahrnehmung der Verantwortlichen, die zu einer neuen Lösung führte.

\subsection{Auswahl}

Ausgehend von einer allgemeinen Vorstellung über Unternehmen im Informationszeitalter (s. Österle 2002) werden eingeführte Lösungen branchenunabhängig für die darin definierten generischen Kooperationsprozesse ausgewählt (s. Tab. 1). Die derzeit siebzehn Praxisfälle aus Europa und den Vereinigten Staaten liefern selbstverständlich keine empirisch abgesicherten Business Networking Konzepte. Sie versuchen aber zu beschreiben, warum die vorgestellten Lösungen erfolgreich waren.

Die sechs generischen Kooperationsprozesse lassen sich dabei auf die Aktivitäten des Kundenprozesses zurückführen (s. Fleisch u. Österle 
2001). Jede Aktivität des Kunden zur Lösung eines Problems wird durch die Verzahnung mit Leistungen vorgelagerter Wertschöpfungspartner effizient unterstützt: Der Kunde informiert sich über Möglichkeiten zur Problemlösung und greift dabei auf das Informationsangebot seiner Partner zurück (Kooperationsprozess Content \& Community). Er spezifiziert seine Anforderungen und wirkt am Design der Lösung mit (Produktlebenszyklus), kauft Produkte und Dienstleistungen ein (Kauf), möchte diese zum richtigen Zeitpunkt am richtigen Ort einsetzen (Logistik), benötigt Unterstützung bei der Instandhaltung der erworbenen Leistung (Instandhaltung) und muss sich um deren Finanzierung kümmern.

Tab. 1. Gegenstand der untersuchten Fälle

\begin{tabular}{|c|c|c|c|}
\hline Kundenaktivität & Kooperationsprozess & Unternehmen & Gegenstand \\
\hline \multirow{3}{*}{$\begin{array}{l}\text { Information und } \\
\text { Evaluation }\end{array}$} & \multirow{3}{*}{ Content\&Communitiy } & Heraeus Kulzer & Multi-Kanal-Management \\
\hline & & Olin Chloralkali & Portal mit bestellbegleitenden Informationen \\
\hline & & Union Investment & gemeinsames Content Management mit Union Polen \\
\hline \multirow{2}{*}{ Design } & \multirow{2}{*}{ Produktlebenszyklus } & ALD/BDK & Verkauf von Finanzierungen über Autohändler \\
\hline & & Brose & elektronischer Anfrageprozess mit Lieferanten \\
\hline \multirow{4}{*}{ Kauf } & \multirow{4}{*}{ Handel (Commerce) } & Boston Scientific & Verbindung ERP-System mit Marktplatz GHX \\
\hline & & Pharma Mall & gemeinsame Internetplattform von 5 Pharmaunternehmen \\
\hline & & Roche Vitamins & B2B-Internet-Shop zum Verkauf von Vitaminen \\
\hline & & Xiameter & Webportal für den Verkauf von Commodity-Produkten \\
\hline \multirow{5}{*}{$\begin{array}{l}\text { Produktion und } \\
\text { Betrieb }\end{array}$} & \multirow{5}{*}{$\begin{array}{l}\text { Logistik } \\
\text { (Supply Chain) }\end{array}$} & L'Oréal & VMI mit dm drogerie markt \\
\hline & & RAG Coal International & Steuerung der Lieferkette für Importkohle \\
\hline & & Röhm & VMI mit BASF Coatings \\
\hline & & Schiesser & Auslagerung der Beschaffungslogistik \\
\hline & & SIG Combibloc & CPFR mit Kunden und Lieferanten \\
\hline \multirow{2}{*}{ Wartung } & \multirow{2}{*}{$\begin{array}{l}\text { Instandhaltung (Main- } \\
\text { tainance \& Repair) }\end{array}$} & ABB Turbosystems & Servicemanagement für Turbolader \\
\hline & & AMAG & Ersatzteilgeschäft für VW, Audi, Porsche etc. \\
\hline Investition & Finanzierung & Aventis & weltweites Investitionscontrolling \\
\hline
\end{tabular}

\section{$4 \quad$ Muster erfolgreicher Kooperation}

\subsection{Eigenschaften erfolgreicher Lösungen}

\subsubsection{Prozesskosten und -beschleunigung}

In allen Fällen werden Reduktionen bei Prozesskosten und Prozessbeschleunigungen als wichtige Nutzenkategorien genannt. Diese werden durch Automatisierung, Auslagerung von Prozessen (Outsourcing) oder einzelnen Prozessschritten (Outtasking), Reduktion von Schnittstellen und/oder Eliminierung von Aufgaben erreicht. Alle lieferantengetriebenen Projekte stellen eine verstärkte Kundenbindung in den Mittelpunkt. Dieser Nutzen wird durch die Individualisierung der angebotenen Leistungen, durch Erhöhung ihrer Verfügbarkeit und das Angebot neuer Services erreicht. 


\subsubsection{Internes ERP als Voraussetzung für Kooperationsprozesse}

Die befragten Unternehmen betonen zudem, dass sie die Kooperationsprozesse nur auf Basis eines integrierten, unternehmensinternen Administrationssystems (ERP) realisieren konnten. (Die Informationsplattform im Fall Schiesser ist beispielsweise direkt mit den SAP R/3-Systemen der Zentrale und der Töchter verbunden.) Die Unternehmen unterstreichen explizit den Vorzug des „Alles-aus-einer-Hand“ gegenüber einem „Best-of-breed“. Hier gilt es allerdings noch, Vergleichsfälle aus Unternehmen mit bewusst heterogenen Softwarearchitekturen zu erheben.

\subsubsection{Start mit 1:1, Vision m:n}

Die Unternehmen haben in ihrer überwiegenden Mehrzahl Lösungen mit einem (1:1) bzw. wie im Fall Schiesser mehreren Partnern (1:n) realisiert. Marktplätze (m:n) haben aus Gründen der Komplexität und aus Angst vor dem Verlust von Wettbewerbsvorteilen durch Prozessstandardisierung bislang offenbar nicht die Bedeutung erlangt, die ihnen in der Theorie beigemessen wird. Die Unternehmen verstehen die realisierten Lösungen aber als einen ersten Schritt in die Richtung einer umfassenden Geschäftsvernetzung und halten sich diese Option für offen.

\subsection{4 „Thing big, start pilot“}

Ein schrittweises Vorgehen und überschaubare Teilprojekte mit konkretem Nutzen sind für Kooperationsprojekte ebenso wie das Change Management und die unternehmensübergreifende Identifikation und Einbindung aller relevanten Interessensgruppen von herausragender Bedeutung. Schiesser reorganisierte zunächst die Aufgabenverteilung zwischen Werken und der Zentrale (Phase 1) und optimierte anschließend die Beschaffungslogistik (Phase 2).

\subsubsection{Entwicklungsrichtungen}

Kooperationslösungen in der Logistik bergen derzeit besonders hohe Potentiale, weil sie den Koordinationsaufwand reduzieren und durch gestraffte Abläufe Liegezeiten von Gütern und Informationen reduzieren. Gleichzeitig wird das Internet im Rahmen einer Multi-Kanal-Strategie zunehmend als zusätzlicher Kanal für den Verkauf und/oder die personalisierte Bereitstellung von verkaufsbegleitenden Informationen (z.B. Rechnung oder Zertifikate) an den Kunden genutzt. Auch die kooperative Produktentwicklung gewinnt langsam an Bedeutung. 


\subsubsection{Kooperationsprozesse bedingen Echtzeitmanagement}

Alle untersuchten Lösungen erhöhen die Prozesseffizienz und schaffen Zusatznutzen für alle Beteiligten. Der Kundennutzen liegt in einer schnelleren, besseren und billigeren Lösung seines Problems. Kooperationsprozesse erreichen dies durch eine engere Verzahnung der Wertschöpfungspartner (bei Schiesser Lieferanten, Produktionswerke, Zentrale und Logistiker) zur gemeinsamen Lösung des Kundenproblems. Voraussetzung für ihre Realisierung ist ein unternehmensübergreifender Datenaustausch, der alle Beteiligten mit den benötigten Informationen auf dem richtigen Aggregationsniveau versorgt - und das ohne Zeitverzögerung. Alle untersuchten Lösungen haben begonnen, dies umzusetzen.

\subsection{Instrumente und Nutzen des Echtzeitmanagements}

Die Echtzeitverbindung zwischen Informationsentstehung und -verwendung führt zu einem Managementkonzept, das das Verständnis für die ITinduzierten Veränderungen der Wirtschaft erleichtert. Echtzeitmanagement bedeutet in einer radikalen Formulierung:

- Jede Information ist sofort nach ihrer Entstehung überall auf dieser Welt verfügbar.

- Jede Aktivität kann alle Informationen dieser Welt ohne Zeitverzug nutzen.

- Von jeder Information kann die Verbindung (Relationship) zu ihrem Kontext hergestellt werden.

Individualisierung, Integration und Automatisierung ermöglichen effiziente Kooperationsprozesse, die schneller, kostengünstiger und flexibler als herkömmliche Kunden-Lieferanten-Beziehungen sind. Zur Realisierung dieser Potentiale nutzen die Unternehmen traditionelle Instrumente des Prozess (Re-)Engineerings (s. z.B. Davenport 1993) und übertragen sie auf die überbetriebliche Ebene: sie automatisieren Aufgaben, lagern einzelne Aufgaben an Dritte aus (Outtasking) und eliminieren unnötige Prozessschritte und (organisatorische wie technische) Schnittstellen. Die Prozessqualität wird durch einen verbesserten Wissensaustausch gesteigert: von aufgabenbezogenen (Dispositions-)Informationen, von Wissen über den aktuellen Zustand des Prozesses (Transparenz) sowie durch den Austausch von Informationen mit anderen Prozessen zur prozessübergreifenden Effizienzsteigerung (z.B. zwischen Angebotsprozess und Vertragsverwaltung). 
Die gesteigerte „Echtzeitigkeit“ in der Zusammenarbeit führt zu Effizienzgewinnen, die beide Partner untereinander aufteilen. Dem Kunden, also dem (End-)Abnehmer der gemeinsam erstellten Leistung entsteht $\mathrm{Zu}$ satznutzen durch einen stärkeren Zuschnitt von Preisen, Leistungen sowie des gesamten Kooperationsprozesses auf seine Bedürfnisse (Individualisierung, One-to-one). Ein vereinfachter Zugang zu den Leistungen wird unterstützt durch:

- die Bündelung der für einen Kundenprozess benötigten Produkte und Dienstleistungen, die dem Kunden die Koordination mehrerer Geschäftsbeziehungen erspart (Everything),

- die Straffung der Prozesse, so dass der Kunden sein Geschäft in einem Vorgang erledigen kann (One-stop),

- das Angebot von Alternativen zur Prozessunterstützung, etwa über verschiedene Kanäle (Anyhow) sowie

- die Möglichkeit für den Kunden, überall und jederzeit Leistungen zu beziehen (Everywhere und Non-stop).

Unmittelbarer Kundennutzen entsteht auch durch eine Aufgabenverlagerung (bei Schiesser z.B. durch die Übernahme der Bestellverfolgung durch Gebrüder Weiss), die Steigerung der Ergebnisqualität (z.B. durch eine automatisierte Meldung von Abweichungen) oder das Angebot neuer Services (z.B. Tracking und Tracing).

Eine zentrale Anlaufstelle für die Partner (One-face-to-the-customer) erhöht die Interaktion zwischen einzelnen Mitarbeitern der beteiligten Unternehmen. Sie erleichtert nicht nur die Ansprache des Kunden oder Lieferanten, sondern ist auch Voraussetzung für das zur gemeinsamen Führung des Kooperationsprozesses notwendige Vertrauen. Die engere Verknüpfung der Partner schafft dem Lieferanten auch dann Nutzen, wenn allein der Kunde von den Effizienzgewinnen durch Kooperationsprozesse profitiert. Einige Unternehmen wie Röhm und L'Oréal können beispielsweise durch Vendor-Managed-Inventory(VMI)-Szenarien die Kundenbindung intensivieren und den Wechselaufwand erhöhen (Customer Lock-in).

\section{Handlungsoptionen}

Die Fallstudien und die daraus abgeleiteten Muster stützen folgende Handlungsoptionen für Unternehmen, welche naturgemäß für das jeweilige wirtschaftliche Umfeld angepasst und konkretisiert werden müssen: 
- Kundensegmentierung nach Kundenprozessen. Eine kundenprozessorientierte Segmentierung unterstützt die Erfassung und Abdeckung von Kundenbedürfnissen.

Das Chemieunternehmen Dow Corning stellt Silikone her. Ein Teil der Kunden möchte innovative Neuentwicklungen einsetzten und sucht dafür umfassende Unterstützung, ein anderer Teil nutzt seit vielen Jahren dieselben Silikone (Commodities) und möchte diese möglichst billig einkaufen. Um den verschiedenen Bedürfnissen gerecht zu werden, bedient Dow Corning Commodity-Käufer jetzt über die Marke Xiameter. Xiameter bietet diesen Kunden die Produkte bei reduziertem Serviceangebot ca. 15\% günstiger als der Markt. Die andere Käufergruppe erhält über die Marke Dow Corning die Produkte ohne Preisabschläge, aberverbunden mit umfassenden Serviceangeboten (Lin u. Senger 2003).

- Verankerung im Tagesgeschäft. Gemeinsam definierte Kooperationsprozesse verankern Strategien im Tagesgeschäft und ermöglichen so ihre Umsetzung. Die Degussa-Tochter Röhm hat beispielsweise mit BASF Coatings ein Konsignationslager realisiert und den zugehörigen Prozess detailliert auf die Besonderheiten dieser Kunden-LieferantenBeziehung zugeschnitten (Österle u. Senger 2003). Xiameter hingegen hat für die Commodity-Kunden einen strikten Bestellprozess definiert, um Kosteneinsparungen mit den Kunden zu teilen. Dadurch sparen Xiameter-Kunden $15 \%$ im Vergleich zu den Marktpreisen, bekommen aber jede Abweichung vom Standardprozess gesondert in Rechnung gestellt (z.B. 250 US\$ bei einer Bestellung per Telefon statt über das Webportal).

- Verteilung des Kooperationsgewinns. Eine langfristig erfolgreiche Kooperation setzt voraus, dass alle Partner davon profitieren. Art und Umfang des Nutzens kann dabei durchaus unterschiedlich sein. Im Fall des Konsignationslagers profitiert Röhm von einer intensivierten Kunden-Lieferanten-Beziehung (Customer Lock-in). BASF Coatings kann die Dispositionsverantwortung an Röhm auslagern und profitiert so durch Zusatznutzen und Kosteneinsparungen. Auch die RAG Coal International, Marktführer beim Import von Steinkohle nach Deutschland, hat bei der Optimierung der Informationsflüsse seiner Lieferkette bewusst ein kooperatives Vorgehen gewählt. Dies soll allen Partnern vom Binnenschiffer bis zu den Umschlaglagern Nutzen stiften und damit die Akzeptanz der entwickelten Lösung „Coal Supply Chain“ sicherstellen (Senger u. Österle 2003a).

- Auswahl der Kooperationslösung. Die existierenden Vernetzungsansätze sind unterschiedlich gut geeignet, um die Umsetzung gewählter 
Strategien zu unterstützen. Kriterien sind die Grösse des Unternehmens und seiner Partner, die bisherige IT-Infrastruktur oder verfügbare Standards.

Portale sind beispielsweise zur Anbindung einer größeren Gruppe von Kooperationspartnern sehr gut geeignet, etwa für die Kunden von Xiameter oder die Partner der RAG Coal International. Portale verursachen jedoch im Regelfall auch Medienbrüche, da die Informationen oftmals aus einem internen Administrationssystem manuell in einen Webbrowser übertragen werden müssen. Die Zusammenarbeit mit wichtigen Partnern wie im Fall Röhm und BASF Coatings kann effizienter über die direkte Kopplung der ERP-Systeme realisiert werden. Die damit verbundenen Investitionen erhöhen gleichzeitig die Wechselkosten der Partner (Customer Lock-in) und damit Intensität und Zeithorizont der Kooperationsbeziehung.

- Durchgängige Softwarearchitektur. Kooperationsprozesse können ihre vollen Potentiale nur entfalten, wenn die Partner in der Lage sind, sie in ihre Back-End-Prozesse und -Systeme zu integrieren. Ein prozessübergreifendes Stammdatenmanagement reduziert die Administrationskosten erheblich. Brose Fahrzeugtechnik GmbH hat beispielsweise ihren Anfrageprozess (Request for quotation, RFQ) „elektronifiziert“. Das Unternehmen entschied sich bewusst gegen die Nutzung eines Marktplatzes, weil dies zum damaligen Zeitpunkt keine Integration mit den vor- und nachgelagerten Prozessen (z.B. Vertragsmanagement) ermöglicht hätte, die in Broses ERP-System abgebildet werden (Österle u. Senger 2003). SIG Combibloc, ein Hersteller von Getränkekartons, hatte eine $\mathrm{CPFR}^{3}$-Lösung mit Lieferanten zunächst prototypisch mit einer vom ERP-System SAP R/3 losgelösten Software implementiert und damit Lagerbestandsreduktionen von bis zu 50\% beim Kunden erreicht. Dennoch entschied sich SIG Combibloc die Lösung mit einer SAPbasierten Software zu realisieren, um u.a. eine redundante Stammdatenhaltung und -pflege zu vermeiden (Senger u. Österle 2003b).

\section{Zusammenfassung}

Die Echtzeitverbindung zwischen Informationsentstehung und -verwendung skizziert ein Managementkonzept, das uns eine Vision des Unternehmens im Informationszeitalter, des Echtzeitunternehmens, vermittelt.

3 Collaborative Planning, Forecasting, and Replenishment, s. www.cpfr.org 
Das Echtzeitunternehmen eng verzahnt mit Kunden und Lieferanten. Unternehmensübergreifende Kooperationsprozesse ermöglichen eine schnellere, bessere und billigere Lösung des Kundenproblems. Sie schaffen dem Kunden Zusatznutzen und verhelfen dem Lieferanten zu einer intensiveren Kundenbindung.

Eine zwischenbetriebliche Kooperation im Stile der heutigen innerbetrieblichen Integration wird allerdings 30 bis 50 Jahre in Anspruch nehmen. Menschen und Unternehmen sind keine Lego-Bausteine, sondern soziale Systeme, welche die Zusammenarbeit langsam erlernen und in Kooperationslösungen verwirklichen müssen. Strategisch operierende Unternehmen investieren aber gerade jetzt in Kooperationsprozesse, realisieren so Nutzen (Quick-wins) und bauen einen Wissens- und Technologievorsprung vor ihren Wettbewerbern auf.

\section{Literaturverzeichnis}

Bauer S, Stickel E (1998): Auswirkungen der Informationstechnologie auf die Entstehung kooperativer Netzwerkorganisationen. Wirtschaftsinformatik Issue $5,434-442$

Davenport TH (1993): Process Innovation: Reengineering Work through Information Technology. Harvard Business School Press, Boston

Fleisch E, Österle H (2001). Vom elektronischen Schaufenster zum Prozessportal - Sieben Thesen zur Gestaltung von erfolgreichen Internetportalen. io Management 4, 38-44

Ives B, Learmonth GP (1984): The Information System as a Competitive Weapon. Communications of the ACM 12: 1193-1201

Lin SE, Senger E (2003) Case Study: Xiameter - e-Commerce Solution Covering Business Customer Ordering and Information Processes. Glassmeyer/McNamee Center for Digital Strategies, Tuck School of Business, Dartmouth College, Hanover, NH, and Institut für Wirtschaftsinformatik, Universität St. Gallen, St. Gallen

Österle H (1995): Business Engineering: Prozess- und Systementwicklung. Springer, Berlin et al.

Österle H (2002): Geschäftsmodell des Informationszeitalters. In: Österle H, Fleisch E, Alt R (Hrsg.) Business Networking in der Praxis: Springer, Berlin, 17-38

Österle H (1995): Business Engineering: Prozess- und Systementwicklung. Springer, Berlin et al.

Österle H, Senger E (2003): Realtime Management - Fünf Fallstudien. Institut für Wirtschaftsinformatik, Universität St. Gallen, St. Gallen

Porter ME, Millar VE. (1985): How Information Gives You Competitive Advantage. Harvard Business Review 4, 149-160 
Prahalad CK, Hamel G (1990): The Core Competence of the Corporation. Harvard Business Review 3 May-June, 79-91

Senger E, Österle H (2002): PROMET BECS - A Project Method for Business Engineering Case Studies.Working Paper BE HSG / BECS / 1, Institut für Wirtschaftsinformatik, Universität St. Gallen, St. Gallen

Senger E, Österle H (2003a): Fallstudie: Transparenz in der ImportkohleLieferkette der RAG; Arbeitsbericht BE HSG / BECS / 16, Institut für Wirtschaftsinformatik, Universität St. Gallen, St. Gallen

Senger E, Österle H (2003b): Fallstudie: Supply Chain Prototyp für Coca-Cola Beverages und SIG Combibloc, Arbeitsbericht BE HSG / BECS / 8, Institut für Wirtschaftsinformatik, Universität St. Gallen, St. Gallen

Senger E (2003c): Fallstudie: Beschaffungslogistik bei der Schiesser AG, Arbeitsbericht BE HSG / BECS / 11, Institut für Wirtschaftsinformatik, Universität St. Gallen, St. Gallen

Stake RE (1995): The Art of Case Study Research. Sage Publications, London

Swatman P, Clarke R (1990): Organisational, Sectoral and International Implications of Electronic Data Interchange, Paper Presented to the 4th IFIP TC-9 World Congress on Human Choices and Computers, Dublin

Yin RK (1994): Case Study Research. Designs and Methods (2 ${ }^{\text {nd }}$ ed.). SAGE Publications, London 